\title{
Histological and Gene Expression Analysis of the Effects of Menopause Status and Hormone Therapy on the Vaginal Introitus and Labia Majora
}

\author{
Robert L. Binder ${ }^{\mathrm{a}}$, Murray A. Freedman ${ }^{\mathrm{b}, \mathrm{c}}$, Kailash B. Sharma ${ }^{\mathrm{b}}$, Miranda A. Farage ${ }^{\mathrm{a}}$, \\ Yu Wanga, Chelsea Combs ${ }^{a}$, David Moore ${ }^{a}$, Jay P. Tiesman ${ }^{a}$, Charles C. Bascomª, \\ Robert J. Isfort ${ }^{\mathrm{a}}$, Raphael Warren ${ }^{\mathrm{a}}$
}

\begin{abstract}
Background: The study aimed to determine the effect of menopausal status and hormone therapy on the introitus and labia majora at the levels of histology and gene expression.

Methods: Three cohorts of 10 women each (pre-menopause, postmenopause and post-menopause + hormone therapy) were selected based on the presentation of clinical atrophy and vaginal $\mathrm{pH}$. Biopsies were obtained from the introitus (fourchette) and labia majora and processed for histology and gene expression analyses with microarrays. Other data collected included self-assessed symptoms, serum estradiol, testosterone, serum hormone binding globulin and the $\mathrm{pH}$ of the vagina and labia majora.
\end{abstract}

Results: The introitus appears exquisitely sensitive to hormone status. Dramatic changes were observed in histology including a thinning of the epithelium in post-menopausal subjects with vaginal atrophy. Furthermore, there was differential expression of many genes that may contribute to tissue remodeling in the atrophic introitus. Levels of expression of genes associated with wound healing, angiogenesis, cell migration/locomotion, dermal structure, apoptosis, inflammation, epithelial cell differentiation, fatty acid, carbohydrate and steroid metabolism were significantly different in the cohort exhibiting atrophy of the introitus. While changes were also observed at the labia, that site was considerably less sensitive to hormone status. The gene expression changes observed at the introitus in this study were very similar to those reported previously in the atrophic vagina providing further evidence that these changes are associated with atrophy.

Conclusions: The histological and gene expression changes occurring within the introitus after menopause may contribute to the constellation of symptoms that constitute the genitourinary syndrome of

Manuscript submitted September 16, 2019, accepted September 27, 2019

${ }^{a}$ The Procter \& Gamble Company, Cincinnati, OH, USA

bobstetrics \& Gynecology, Medical College of Georgia, Augusta, GA, USA ${ }^{\mathrm{c} C o r r e s p o n d i n g ~ A u t h o r: ~ M u r r a y ~ A . ~ F r e e d m a n, ~ O b s t e t r i c s ~ \& ~ G y n e c o l o g y, ~}$ Medical College of Georgia, Suite 4100, 1348 Walton Way, Augusta, GA 30901, USA. Email: mfreedmd@knology.net

doi: https://doi.org/10.14740/jocmr4006 menopause.

Keywords: Menopause; Atrophy; Introitus; Labia majora; Vagina; Histology; Gene expression

\section{Introduction}

Menopause is associated with a dramatic decline in circulating estradiol [1]. Because of the high concentration of estrogen receptors in the urogenital tract, the hypoestrogenic state contributes to the progressive atrophy of tissues in this area of the body. This involution includes a contraction and loss of elasticity at the introitus, a shorter and narrower vestibule having fewer vaginal folds or rugae, and a thinner and more friable vaginal mucosa that is more sensitive to proprioception and easily traumatized [2]. Distal to the vagina, the labia majora and labia minora become thinner and shrink, hair becomes sparse and the labia often lose their distinguishing features and may actually fuse [1]. The primary surrogate measures associated with hypoestrogenicity in the urogenital tissues include a rise in vaginal $\mathrm{pH}$ to greater than 5 and a reduction in the vaginal maturation index (VMI) [3, 4]. While most of the exfoliated cells in the pre-menopausal vagina are glycogen-rich superficial cells, the decline in estrogen is associated with a reduced number of these cells and a corresponding increase in parabasal cells. This loss of glycogen coupled with a reduction in acid production contributes to the change in $\mathrm{pH}$ and microbiome $[5,6]$. Although the predominant post-menopausal symptoms are vaginal dryness and painful intercourse (dyspareunia), the early clinical manifestations are more pronounced at the more distal region of the vagina, i.e. the introitus (fourchette), rather than the proximal or upper vagina [2]. The anatomic and cellular changes along with the resultant symptoms are now referred to collectively as the genitourinary syndrome of menopause (GSM) [7]. With life expectancy now extending into the $80 \mathrm{~s}$, a large percentage of women can be expected to experience considerable symptomatology and a compromise in their quality of life [8-10].

While the current standard of care for women with GSM is systemic or local vaginal estrogen therapy (ET), there is public 
concern and confusion regarding the effects of long-term use of hormone therapy (HT). Development of non-hormonal alternatives would be of value and facilitated by the identification of the molecular pathways associated with estrogen treatment. One approach is to identify the genes in urogenital tissues effected by ET. A previous study reported the effects of 12 weeks of systemic ET on vaginal gene expression profiles in women who had undergone hysterectomy and were defined as atrophic by low serum estrogen, high vaginal $\mathrm{pH}$ and low VMI scores $[11,12]$. Among the regulated processes of interest reported were tissue repair and remodeling. Another study focused on post-menopausal women with and without vaginal dryness and assessed the vaginal microbiome and gene expression in vaginal epithelium [13]. Moderate to severe dryness was associated with a decrease in the Lactobacillus, increased microbial diversity and gene expression changes related to inflammation and epithelial differentiation. Similarly, a recent transcriptomic study of vaginal tissue compared two post-menopausal groups of women: breast cancer patients on an aromatase inhibitor (AI) with markedly depressed estrogen levels versus healthy women using vaginal ET [14]. The dominant gene expression changes observed were related to decreased epithelial differentiation in the AI-treated women. To improve our understanding of how HT status effects urogenital tissue associated with atrophic symptoms, we compared the gene expression profiles and histology of the introitus and labia majora in three cohorts of women: 1) 10 normal pre-menopausal; 2) 10 hypoestrogenic post-menopausal; and 3) 10 estrogen replete post-menopausal on HT. The introitus was selected as a site extremely sensitive to estrogen and the labia was selected as the site representative of epithelial tissue immediately adjacent to the vagina. The data collected in the study included symptoms, along with $\mathrm{pH}$, clinical atrophy assessment, histological features from hematoxylin/eosin (H\&E)-stained sections and global gene expression profiles from microarray analyses. We then compared the vaginal gene expression profiles derived from the earlier HT study $[11,12]$ with our data set to identify potential similarities and differences.

\section{Materials and Methods}

\section{Study population}

This study was conducted at University Hospital (Augusta, GA). The University Hospital Institutional Review Board approved the protocol and informed consent.

\section{Study design}

Briefly, three cohorts of 10 healthy women each were selected for this study and included: 1) Pre-menopausal women showing little to no sign of clinical urogenital atrophy and having a vaginal $\mathrm{pH}<5.0$ (Pre-M); 2) Post-menopausal women with signs of clinical urogenital atrophy and having a vaginal $\mathrm{pH} \geq$ 5.0 (Post-M); and 3) Post-menopausal women on systemic HT with little or no sign of clinical urogenital atrophy and having a vaginal $\mathrm{pH}<5.0$ (Post-M + HT).

\section{Health status}

A medical questionnaire was used to establish the general health of all subjects. All women were free of vulvar pathology or lesions (e.g. cuts, lesions, rash, body piercing, etc.); refrained from douching and did not use aspirin or consume alcohol or a caffeinated beverage within $48 \mathrm{~h}$ of the biopsy procedure; and had refrained from sexual intercourse or application of perfumes or cosmetics to the genital area within $24 \mathrm{~h}$ of the biopsy procedure. All women were asked to clip a small area of their labia majora to be free of hair prior to the biopsy procedure. Women were excluded if they had uncontrolled urinary incontinence and/or a medical condition existed that in the opinion of the investigator (MA Freedman) would preclude participation. Pre-M women were 21 - 45 years old, had an intact uterus and regular menstrual cycles ( 21 - 35 days), and had not been pregnant within 6 months of the study or currently nursing. All Post-M women were 55 - 70 years old and had not had a menstrual period for at least 1 year. All medications were recorded, including the length of time medicated with HT. HT administration was oral, transdermal or injectable and five subjects in the Post-M + HT group received concomitant testosterone therapy. Use of oral contraceptives (in the Pre-M group) was recorded but not restricted.

\section{Clinical assessment of urogenital atrophy}

Subjects were examined, and the following parameters were evaluated: 1) Rugosity at the hymeneal area; 2) Morphology of the vestibule; 3) Elasticity (stenosis) at the introitus; 4) Patulousness or telescoping urethra; and 5) Color of the epithelium (pallor, friability, petechiae). Each parameter was graded on scale of none (0), minimal/mild (1), or marked/severe (2). A collective score $\leq 2$ was considered normal (non-atrophic) and a score $\geq 6$ was considered atrophic. Vaginal $\mathrm{pH}$ was measured as described previously using $\mathrm{pH}$ paper [2]. Vulvar $\mathrm{pH}$ was obtained using a flathead pH probe (Skincheck HI98109; Hanna Instruments, Ann Arbor, MI).

\section{Biopsy collection and histology}

The skin surface was cleansed with Betadine ${ }^{\circledR}$ and the areas to be biopsied were then anesthetized with lidocaine. After the anesthetic had been administered, the surface was cleansed with alcohol. A Tischler-Morgan ${ }^{\mathrm{TM}}$ punch biopsy instrument (Cooper Surgical 64-689; Trumbull, CT) was used to obtain a biopsy from the introitus at the level of the hymeneal carunculae and another from the midpoint of the labia majora adjacent to the introitus; both biopsy sites were reapproximated with a chromic suture. Importantly, the biopsy instrument insured a uniform biopsy thickness. The side of the labia (left or right) selected for the biopsy was randomized. Each biopsy was bisected sharply across the epithelial-dermal axis. One half was prepared for microarray analysis and the remaining half was prepared for histological examination.

The histological specimens were immersed overnight in 
$10 \%$ neutral buffered formalin, then transferred into $70 \%$ ethanol paraffin embedded, sectioning at $4 \mu \mathrm{m}$ and stained with H\&E. Images were obtained using a Nikon Eclipse E400 microscope equipped with Nikon Digital Camera DXM1200F with $\times 0.7$ DXN lens and $\times 10$ objective. As a measure of the abundance of rete ridges, which are projections of epithelium into the papillary dermis, H\&E-stained sections were evaluated for the degree of undulation of the dermal-epithelial interface using image analysis. Specifically, the lengths of the epithelialdermal interface and epithelial surface were measured across each section. The ratio of these values (interface/surface) indicates the degree undulation of epithelial-dermal boundary.

\section{Microarray analysis}

Biopsy specimens for microarray analysis were first immersed in RNALater (ThermoFisher Scientific, Waltham, MA) at room temperature, transferred into phosphate-buffered saline, and finally transferred into a 1.5-mL Eppendorf tube and snap frozen in a dry ice-ethanol bath before storage at -70 to -80 ${ }^{\circ} \mathrm{C}$. The RNA was extracted and purified using an RNeasy kit (QIAgen, Valencia, CA). The purified RNA was converted to biotin-labeled cRNA copies using the Affymetrix HT 3' IVT Express kit (Santa Clara, CA) according to the manufacturer's protocol and executed on a Beckman Biomek ${ }^{\circledR}$ FX $^{\mathrm{p}}$ Laboratory Automation Workstation (Indianapolis, IN). Biotinylated cRNA was fragmented by limited alkaline hydrolysis and then hybridized overnight to Affymetrix GeneTitan ${ }^{\circledR}$ U219 array plates using the Affymetrix GeneTitan ${ }^{\circledR}$ instrument. Following processing, chip images were converted to numeric data using the PLIER algorithm as executed in the Affymetrix GeneChip Expression Console followed by statistical QC analysis to confirm data quality. Outliers were removed from further analysis. After QC analysis, all groups had 10 samples except the Pre-M and Post-M labia majora groups, which each had nine samples.

Microarray data from the published ET treatment study of vaginal atrophy $[11,12]$ were downloaded from the GEO Database [15] (accession number GSE11622) for comparison. In that study, RNA isolated from the biopsies was subjected to gene expression profiling on Affymetrix HG-U133 Plus 2.0 GeneChips. We reanalyzed the raw CEL file data obtained from GEO, and the data were summarized and normalized using Affymetrix MAS5.0 software.

Two-sample $t$-tests methods were used to examine differences among the three groups separately for the introitus and labia majora. This was conducted one probe set at a time. Paired $t$-tests were performed to examine the difference between baseline and after ET for the vaginal data. The Benjamini-Hochberg procedure was used to calculate false discovery rate (FDR) and q-values (adjusted $\mathrm{P}$ values) to get an indication of the level of false positives within the significant results [16]. Expression data were summarized in heat maps as $\log _{2}$ fold changes and in tables as signed fold changes. These were calculated as the simple ratio of signal values $(\mathrm{A} / \mathrm{B})$ for positive changes and $(-1 /(\mathrm{A} / \mathrm{B}))$ for decreasing changes $(\mathrm{A}<\mathrm{B})$, which makes up- and down-regulated gene expression changes symmetrical on a linear scale.

Probe set lists from the introitus and labia majora were filtered for the top $70 \%$ by maximum mean signal across the groups to remove transcripts that may not be expressed in the sampled tissues. Various filtering for statistical significance and fold change was done as described in the tables and figure legends. Filtered probe set lists were subject to hierarchical clustering to visualize global patterns using OmniViz Version 6.1.4 software (Instem Scientific, Cambridge, UK) with the Euclidean similarity metric. OmniViz was also used to identify probe sets associated with collagen biology by text queries of gene ontology (GO) biological process annotation downloaded from the Gene Ontology Consortium [17]. The transcripts of many genes have more than one complementary Affymetrix probe set. In the tables with gene list summaries only one probe set per gene is displayed for conciseness. These were selected by inspection giving preference to the HG-U219 probe sets with the suffixes at and_a_at, which are most specific, and then to the most highly statistically significant probe set for a given gene. All the multiple HG-U219 probe sets gave consistent and similar results for the genes reported here. There was no apparent effect of medications (testosterone, progestin, or contraceptives) on gene expression patterns, although our sampling was small.

GO term enrichment analysis to identify regulated biological processes was performed using the DAVID (version 6.7) Bioinformatics Resources functional annotation clustering tool with Entrez Gene IDs [18, 19]. The analyses were done at medium stringency, and the GO FAT biological process category that filters out very broad, non-specific GO terms was used. The up- and down-regulated gene lists were filtered as described in the table legends and the background gene lists consisted of the top $70 \%$ of probe sets based on maximum mean signal values without statistical filtering.

The published vaginal atrophy study $[11,12]$ data were compared to the current study using cross GeneChip model mapping files provided by Affymetrix. For the most stringent comparison, the Best Match was used. Data from the vaginal atrophy study, which used the HG-133 plus 2.0 GeneChip, were filtered for $>10 \%$ Present calls from the MAS5.0 summary and the HG-U219 data (current study) were filtered for signal as described above. For the cross-study comparison matches were given preference in the order Best Match, Good Match and then by gene identifier. In the case of redundant HG-U133 Plus 2.0 probe sets, the probe sets displayed were selected based on the quality of the match, the highest number of Present calls and then by statistical significance. Fold changes for the vaginal data were calculated using the average group data, rather than paired results, to be more comparable to the current study.

\section{Other measures}

Each subject completed a questionnaire that queried presence and magnitude of symptoms associated with urogenital atrophy. These included vaginal dryness, vulvar and/or vaginal itching, and dyspareunia. Symptoms were rated on a scale of none (0), slight (1), moderate (2), considerable (3) and unbearable (4). Blood samples for analysis of estradiol, testosterone and serum hormone binding globulin (SHBG) were obtained from each subject at the time of biopsy. SHBG was quantified using an electrochemiluminescence immunoassay (Elecsys ${ }^{\circledR}$ 
Table 1. Subject Attributes

\begin{tabular}{|c|c|c|c|c|c|}
\hline Attribute & Pre-M & $\begin{array}{l}\text { P value of Pre-M } \\
\text { vs. Post-M }\end{array}$ & Post-M & $\begin{array}{l}\text { P value of Post-M } \\
\text { vs. Post-M + HT }\end{array}$ & Post-M + HT \\
\hline Mean age & $30.5 \pm 2.1$ & & $62.9 \pm 1.1$ & & $59.5 \pm 1.7$ \\
\hline Years on HT & NA & & NA & & $15.7 \pm 2.2$ \\
\hline Clinical atrophy grade $(0-10)$ & 0 & $<0.0001$ & $8.1 \pm 0.4$ & $<0.0001$ & $0.6 \pm 0.3$ \\
\hline Vaginal $\mathrm{pH}$ & $4.2 \pm 0.1$ & $<0.0002$ & $5.4 \pm 0.1$ & $<0.0002$ & $4.1 \pm 0.1$ \\
\hline SHBG $(\mathrm{nmol} / \mathrm{L})$ & $104 \pm 22$ & & $67 \pm 12$ & & $82 \pm 16$ \\
\hline Testosterone (ng/dL) & $30 \pm 5$ & & $20 \pm 4$ & $<0.009$ & $89 \pm 19$ \\
\hline
\end{tabular}

$\mathrm{N}=10$ per group. Data are mean \pm SE. Pre-M: pre-menopause; Post-M: post-menopause; NA: not applicable; SE: standard error; HT: hormone therapy; SHBG: serum hormone binding globulin.

SHBG) with the Roche Diagnostics Elecsys ${ }^{\circledR} 2010$ Analyzer (Roche Diagnostics, Switzerland).

Statistical analyses including (but were not limited to) $t$ tests, analysis of variance (ANOVA) and multivariate methods were conducted to examine differences among different groups. A one-way ANOVA model with terms for groups (Pre$\mathrm{M}$, Post-M and Post-M + HT) was constructed to test the group differences. This was performed respectively for the clinical atrophy score, vaginal $\mathrm{pH}$ and vulvar $\mathrm{pH}$. The three possible pair wise group comparison results were extracted from the ANOVA model. Measures of association (Pearson correlation method) were used to evaluate the relationship between $\mathrm{pH}$ values and clinical atrophy score, and the $\mathrm{pH}$ values relationship between the vagina and the vulva. Non-parametric tests (Fisher's exact test and Kruskal-Wallis one-way ANOVA) were used to test the differences among the groups for symptom scores (e.g. dryness, itch, etc.)

\section{Results}

\section{Enrollment}

The results of the clinical atrophy grades and vaginal $\mathrm{pH}$ were commensurate with the screening criteria used to enroll the subjects (Table 1). The "low" mean serum estradiol level in the Pre-M cohort reflects the use of oral contraceptive (six of 10 subjects) and the "high" mean serum testosterone level in the Post-M + HT cohort reflects testosterone use (five of the 10 subjects). While there was a large shift in vaginal $\mathrm{pH}$ comparing Post-M to either Pre-M or Post-M + HT, there was a smaller $\mathrm{pH}$ shift at the labia. The two symptoms most affected by HT status (Fig. 1) were vaginal dryness $(\mathrm{P}<0.005)$ and dyspareunia, which presented to a lesser extent in the women on HT.

\section{Histology changes}

H\&E-stained sections of biopsied tissue from the introitus revealed structural changes in the Post-M group similar to those reported in the more upper vagina, such as the loss of superficial epithelial layers and epithelial thinning (Fig. 2) [3, 20-22]. Equally pronounced was the loss of rete ridges or undulations of the epithelial-dermal interface (Fig. 2a-c). These changes were quantified using image analysis as the ratio of the lengths of the undulating epithelial-dermal junction to the flatter epithelial surface. For the introitus, the ratios were Pre-M: 1.67 \pm 0.48 , Post-M: $1.28 \pm 0.33$, Post-M + HT: $1.98 \pm 0.97$ (all \pm standard deviation (SD)). The comparisons of Pre-M to Post$\mathrm{M}$ and Post-M to Post-M + HT groups were significant at $\mathrm{P}<$ 0.05 , with no significant difference between Pre-M and Post$\mathrm{M}+\mathrm{HT}$ groups. The rete ridge changes at the labia majora between Pre-M and Post-M were directional different but nonsignificant.

\section{Microarray analysis}

There were very substantial changes in gene expression in the introitus associated with atrophy. The GeneChip model used for the analysis contains 49,293 probe sets complementary to the transcripts of more than 20,000 genes. For the Post-M to Pre-M comparison, 5,594 probe sets met an FDR adjusted P value of $\mathrm{q}<0.05$. In contrast, no probe sets met this highly stringent statistical filtering in the labia majora. Hierarchical clustering (Fig. 3) was performed to visualize the patterns of differential gene expression for the various group comparisons using somewhat less stringent statistical filtering. The probe sets displayed in the heat maps were selected separately for introitus (Fig. 3a) or labia majora (Fig. 3b). For each site probe sets were selected for unadjusted $\mathrm{P}<0.01$ in either the Post$\mathrm{M}$ to Pre-M or the Post-M to Post-M + HT comparisons (i.e. atrophied to non-atrophied). Additional filtering was done to eliminate probe sets with low signal as described in "Methods". This filtering yielded a total of 7,488 probe sets for the introitus. The patterns of differential gene expression for the Post-M to Pre-M and Post-M to Post-M + HT introitus comparisons were remarkably similar (first two columns in Fig. 3a). Additionally, the Venn diagrams (Fig. 3c) show the quantitative relationships between the numbers of probe sets that show directional consistency. There was approximately $99 \%$ 


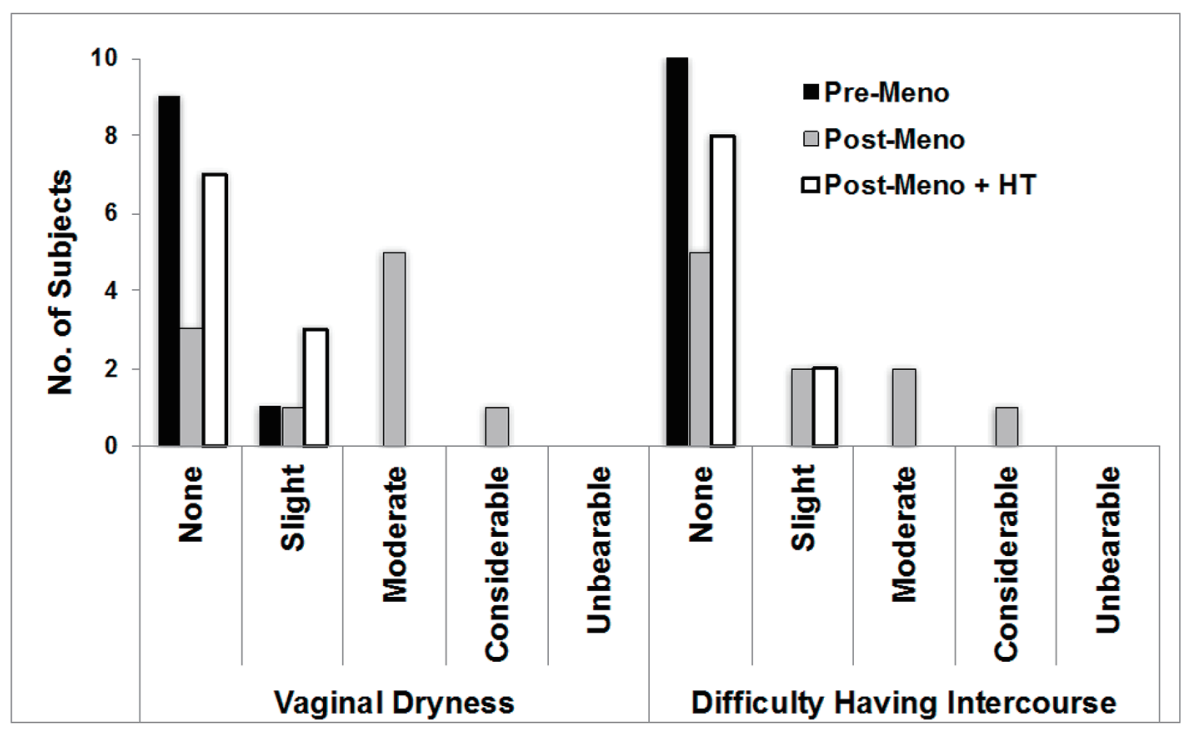

Figure 1. Urogenital symptoms. Shown are the symptoms that differed significantly across the study cohorts. ANOVA group effects: vaginal dryness $(P=0.003)$ and difficulty having intercourse $(P=0.02)$. ANOVA: analysis of variance; Pre-M: pre-menopause; Post-M: post-menopause; HT: hormone therapy.

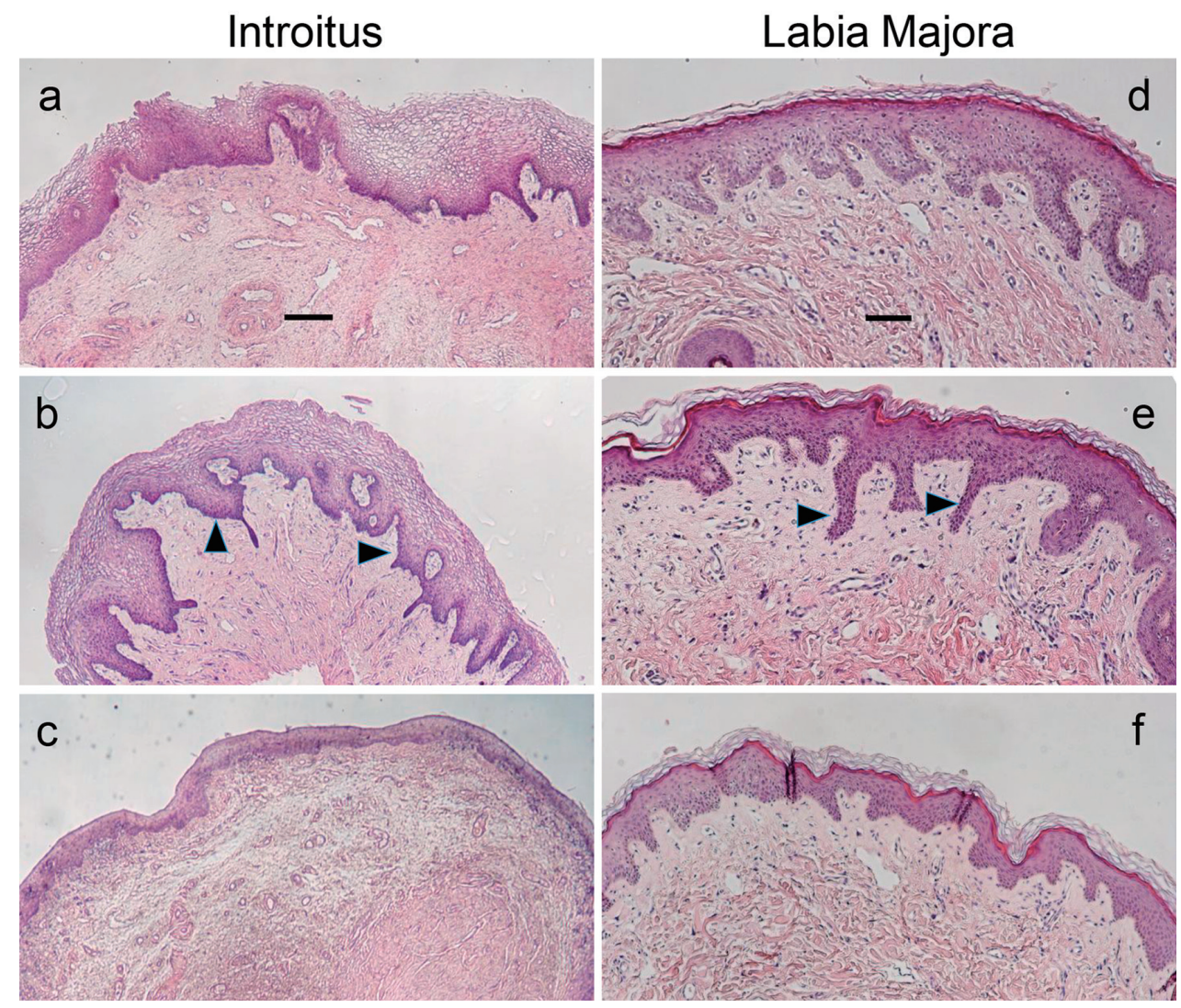

Figure 2. H\&E-stained introitus and labia majora tissues. Histological sections of biopsies obtained from the introitus (a-c, scale bar $=200 \mu \mathrm{m})$ and labia majora $(\mathrm{d}-\mathrm{f}$, scale bar $=100 \mu \mathrm{m})$ representative of the three study cohorts. The same subject was the source for each site pair. (a) and (d): pre-menopause; (b) and (e): post-menopause + HT; (c) and (f): post-menopause. Arrows point to examples of rete ridges or projections of the epithelium into the dermis. Note the reduced thickness of the epithelium and abundance of rete ridges in the post-menopausal group. Lower magnification images were used for the introitus to illustrate the differences across the cohorts more clearly. H\&E: hematoxylin/eosin; HT: hormone therapy. 
Introitus
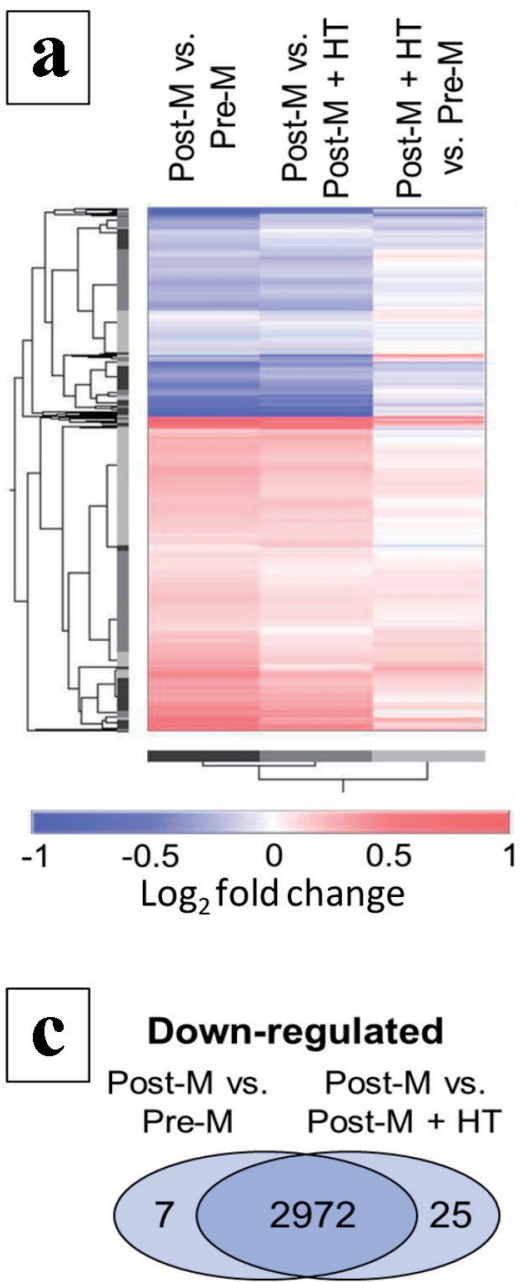

$99.3 \%$ concordance

\section{Up-regulated}

Post-M vs. Post-M vs. Pre-M Post-M + HT

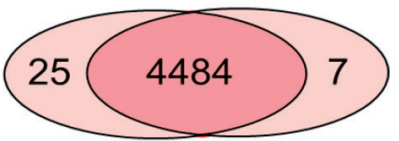

$98.9 \%$ concordance

\section{Labia Majora}
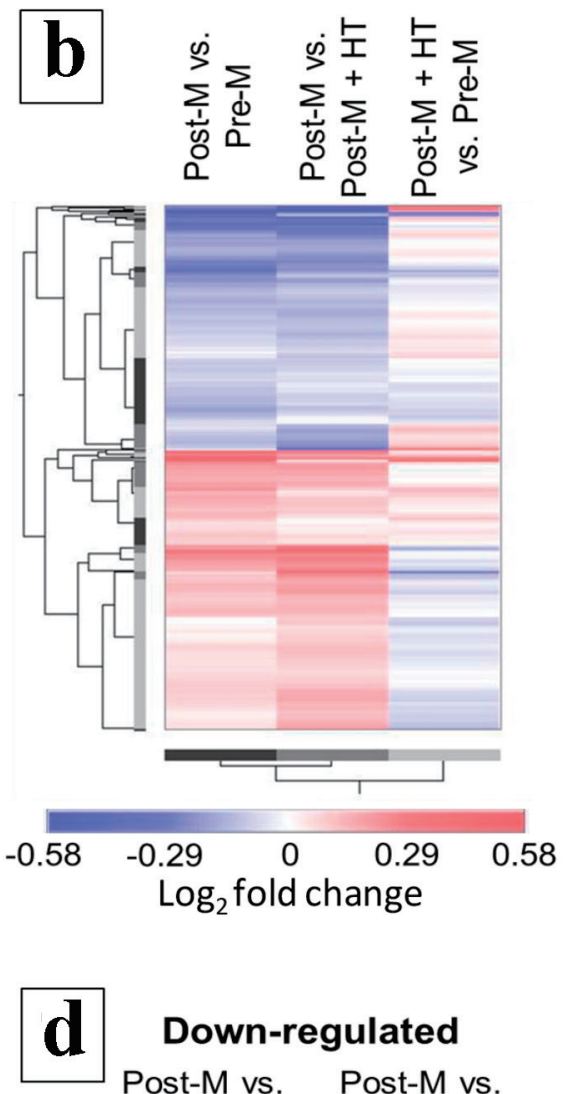

Down-regulated

Post-M vs. Post-M vs.

Pre-M Post-M + HT

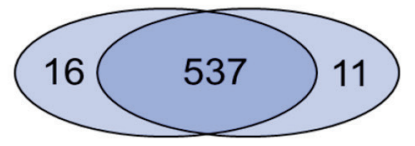

95.2\% concordance

\section{Up-regulated}

Post-M vs. Post-M vs.

Pre-M Post-M + HT

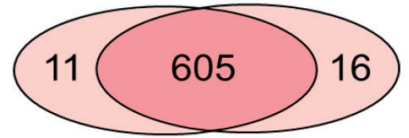

95.7\% concordance

Figure 3. Hierarchical clustering of differentially regulated gene transcripts. (a) and (c) refer to the introitus and (b) and (d) refer to labia majora. (a) and (b) show probe sets differentially regulated in Post-M tissue. Probe sets were filtered for $P<0.01$ by $t$-test for the group comparisons Post-M to Pre-M or Post-M to Post-M + HT. Other data filtering is described in "Methods". The results of the Post-M + HT to Pre-M comparison is shown but was not used in the data filtering. The data are presented as $\log _{2}$ fold changes with red indicating up-regulation and blue down-regulation. The color ramps indicate the scales with $\log _{2}$ values shown. (c) and (d) show the directional consistency of the differential gene expression patterns in the Post-M to Pre-M and Post-M to Post-M + HT comparisons displayed as Venn diagrams. Pre-M: pre-menopause; Post-M: post-menopause; HT: hormone therapy.

concordance between the directional patterns of gene expression in the Post-M comparisons to the Pre-M and Post-M + HT groups (up- and down-regulation). These results are consistent with the fact that the women on HT did not have signs and symptoms of vaginal atrophy and presented with normal histology. However, in the introitus, many of the post-menopausal 
changes in gene expression were possibly weakly evident in the group on HT, since the pattern of differential gene expression in Post-M + HT to Pre-M groups showed similarity to the Post-M to Pre-M comparison (compare the first and last columns in Fig. 3a). There was $82 \%$ directional concordance of the $\log _{2}$ fold changes for these two comparisons.

A similar pattern was seen in the labia majora for the Post$\mathrm{M}$ comparisons to the Pre-M and Post-M + HT groups, but only 1,169 probe sets met the statistical filtering criteria (Fig. $3 b$ and d). Note also that the $\log _{2}$ fold change color ramp ranges in Figure 3a and $b$ are different reflecting that the magnitude of differential gene expression in the various comparisons was generally smaller in the labia majora. The Venn diagrams in Figure $3 \mathrm{~d}$ reveal that for the labia majora, there was $>95 \%$ directional concordance in differential gene expression patterns for the Post-M comparisons to the Pre-M and Post-M + HT groups. Unlike the introitus, the Post-M + HT to Pre-M and Post-M to Pre-M comparisons were not very similar (compare the first and last columns in Figure 3b, which have only about $43 \%$ directional concordance). The overall analyses for both the introitus and labia majora show that, compared to postmenopausal subjects with vaginal atrophy, the subjects on HT (and without vaginal atrophy) had global patterns of differential gene expression closely resembling those of the younger pre-menopausal subjects, although there are quantitative differences in gene expression between the groups.

Enrichment analysis for GO terms and other sources of gene annotation is a common approach to identify biological patterns in large sets of differentially expressed genes. Table 2 shows summary results for GO biological process enrichment analysis for the genes up- and down-regulated in the atrophic introitus. The analysis algorithm used clustered related terms with many genes in common and only the most statistically significant term for each cluster is shown. There is a common thread among the top up-regulated processes related to response to wounding, angiogenesis, cell migration/locomotion and immune regulation. Among the down-regulated biological processes was "epithelial cell differentiation", consistent with the thinning epithelium (Fig. 2). The other down-regulated biological processes all relate to metabolism including fatty acid, steroid, hormone and carbohydrate metabolism. A separate analysis using labia major data, which had far fewer significant genes, gave more limited results (not shown). However, for the Post-M to Pre-M labia majora group comparison, the most significantly enriched terms were "immune response" (up-regulated, $\mathrm{P}=9 \times 10^{-5}$ ) and "collagen metabolic process" (down-regulated, $\mathrm{P}=2 \times 10^{-4}$ ).

A stringent cross-body site comparison was performed to identify genes commonly regulated in both the introitus and labia majora with urogenital atrophy. The gene list was filtered as described in Table 3 for fold change and statistical significance in all the comparisons shown. Only eight genes met the filtering criteria and all of them showed the same directional regulation in both tissues, although there was no filtering for directionality. The down-regulated genes were GREB1, EHF, TP63, DIO2, IGF1 and RBM47, and the up-regulated genes were MFAP4 and $C 3$. These consistently regulated genes play fundamental roles in the maintenance of normal cellular and tissue function and innate immunity (see "Discussion").

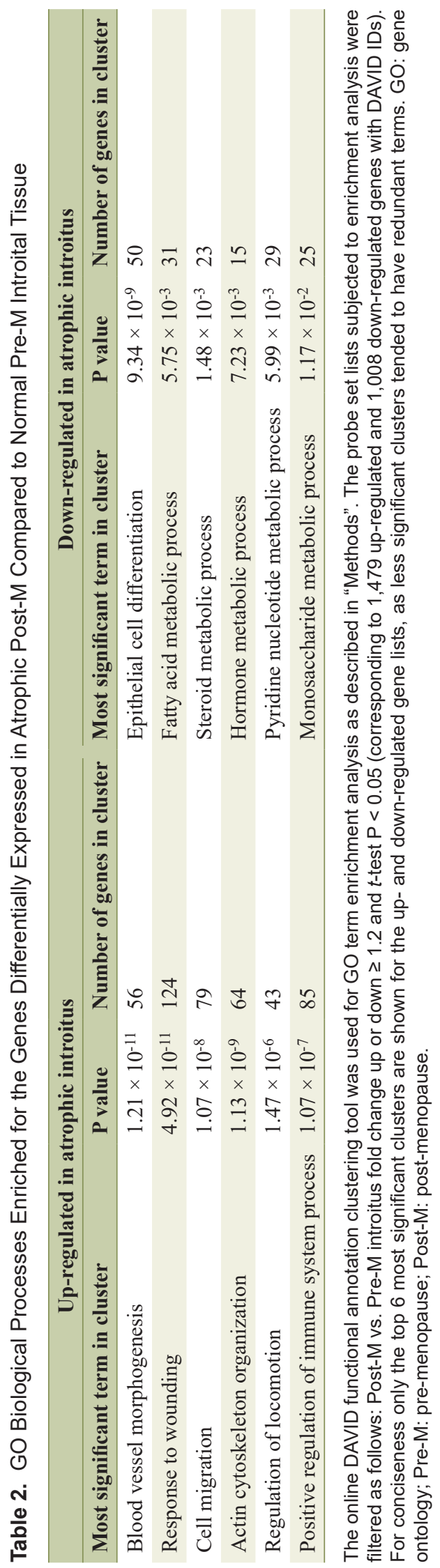




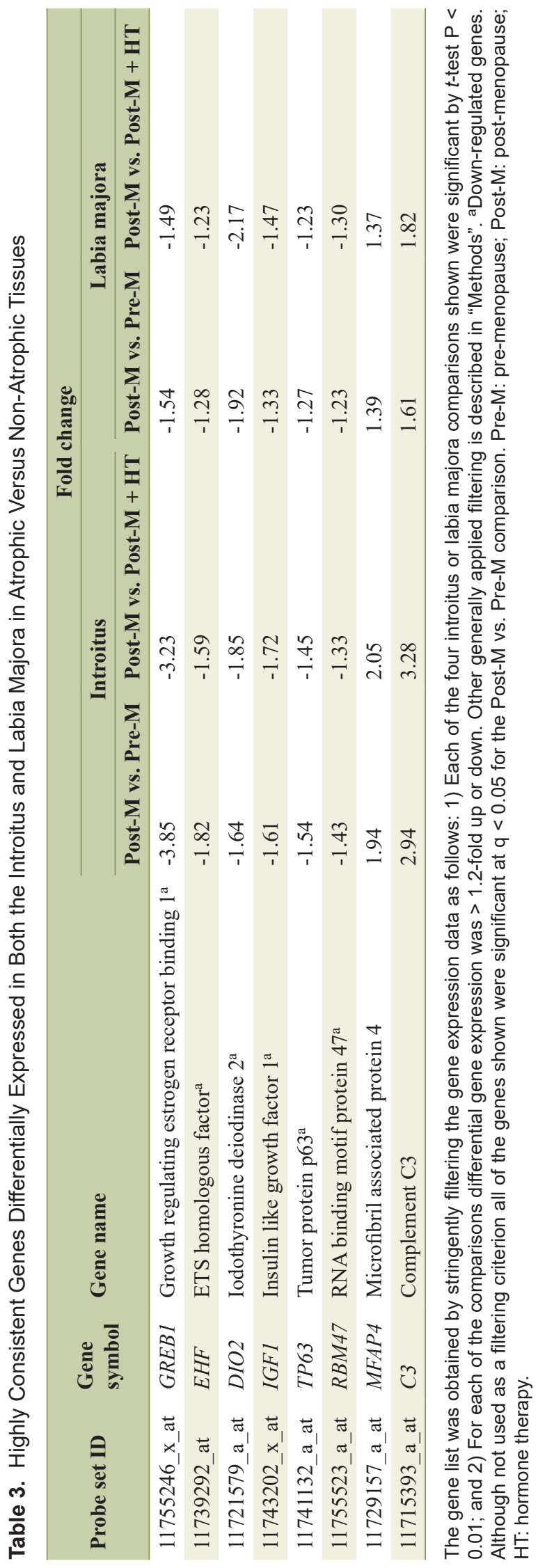

Atrophy typically involves loss of tissue mass. Since collagens form a major fraction of the dermal compartment, we queried the data to identify significantly regulated genes annotated with the GO term "collagen fibril organization" (Table 4). Expression of each of the following major structural collagens, COL1A1, COL1A2 and COL3A1, and the low abundance collagen, $C O L 5 A 2$, was significantly down-regulated in the Post$\mathrm{M}$ atrophied introitus. Lysyl oxidase $(L O X)$, which codes for an enzyme that modifies collagen and elastin leading to interpolypeptide chain cross links, and which appears necessary for proper collagen fibril organization [23], was also significantly down-regulated. In contrast, lysyl hydroxylase 3 (PLOD3), which codes for another collagen processing enzyme that hydroxylates lysines and attaches sugars necessary for proper fibril organization [24], was modestly up-regulated. The genes for two extracellular matrix components that interact with collagens and play a role in fibril formation, lumican (LUM) and dermatopontin $(D P T)$ [25-27] were strongly up-regulated with atrophy. Secreted frizzled-related protein 2 (SFRP2), a member of the SFRP family that modulates WNT pathway signaling [28], was up-regulated more than twofold with atrophy in the introitus. COL1A1,COL1A2 and COL3A1,COL5A2 and $L U M$ showed a similar statistically significant regulation $(\mathrm{P}<$ 0.05 ) in the labia majora (Post-M to Pre-M), although the effects were less strong than the introitus (data not shown).

A comparison was conducted between the current study and an earlier published study of vaginal atrophy [11, 12]. Figure 4 shows the patterns of differential expression of transcripts stringently filtered for adjusted $P$ values $(q<0.05)$ in both the introitus Post-M vs. Pre-M and vagina baseline vs. ET comparisons. Of the probe sets meeting the statistical filtering, there were 734 from the HG-U219 GeneChip (current study) that mapped to 654 on the HG-U133 Plus 2.0 model (vaginal study), and these represent transcripts of 589 genes. The patterns of differential gene expression in atrophied versus non-atrophied tissues were remarkably similar for the introitus and vagina. Of the 589 genes, only two showed directionally different patterns (up in vagina and down in introitus: KANK1 (KN motif and ankyrin repeat domains 1); down in vagina up in introitus: HNRNPAO (heterogeneous nuclear ribonucleoprotein $A 0)$ ). Although the $\log _{2}$ fold changes for many transcripts appear higher in the vagina, direct quantitative comparisons between the two experiments may not be appropriate because the two GeneChip models have different designs, the data were normalized with different algorithms and tissue sampling may have been different.

GO term enrichment analysis was performed on the genes filtered for common regulation in introital and vaginal atrophy (Table 5). The results show similarity to those obtained in the analysis of the introitus data (Table 2), although there were substantially fewer genes in the more stringently filtered crossstudy list. The analysis revealed up-regulation of immune response-related processes, cytoskeleton gene expression and apoptosis, and down-regulation of epithelial differentiation and carbohydrate metabolism in the atrophic tissues. Phospholipid metabolic processes were also down-regulated, consistent with the themes of down-regulation of fatty acid and steroid metabolic processes identified in the separate introital data analysis (Table 2). Furthermore, expression of several genes associated 

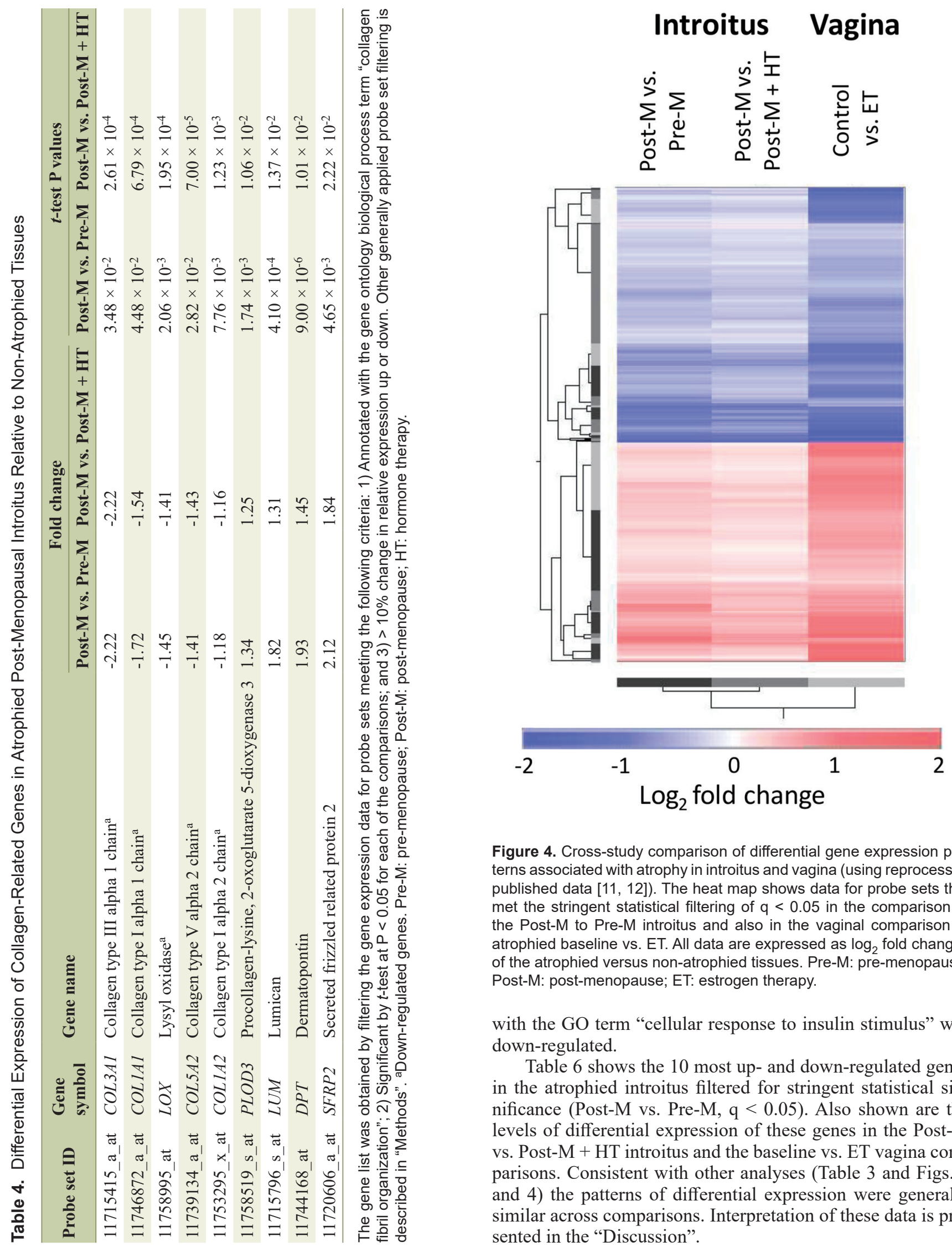

Figure 4. Cross-study comparison of differential gene expression patterns associated with atrophy in introitus and vagina (using reprocessed published data [11, 12]). The heat map shows data for probe sets that met the stringent statistical filtering of $q<0.05$ in the comparison of the Post-M to Pre-M introitus and also in the vaginal comparison of atrophied baseline vs. ET. All data are expressed as $\log _{2}$ fold changes of the atrophied versus non-atrophied tissues. Pre-M: pre-menopause; Post-M: post-menopause; ET: estrogen therapy.

with the GO term "cellular response to insulin stimulus" was down-regulated.

Table 6 shows the 10 most up- and down-regulated genes in the atrophied introitus filtered for stringent statistical significance (Post-M vs. Pre-M, q < 0.05). Also shown are the levels of differential expression of these genes in the Post-M vs. Post-M + HT introitus and the baseline vs. ET vagina comparisons. Consistent with other analyses (Table 3 and Figs. 3 and 4) the patterns of differential expression were generally similar across comparisons. Interpretation of these data is presented in the "Discussion". 
Table 5. GO Biological Processes Enriched for Genes Commonly Regulated in Atrophied Vaginal and Introital Tissues

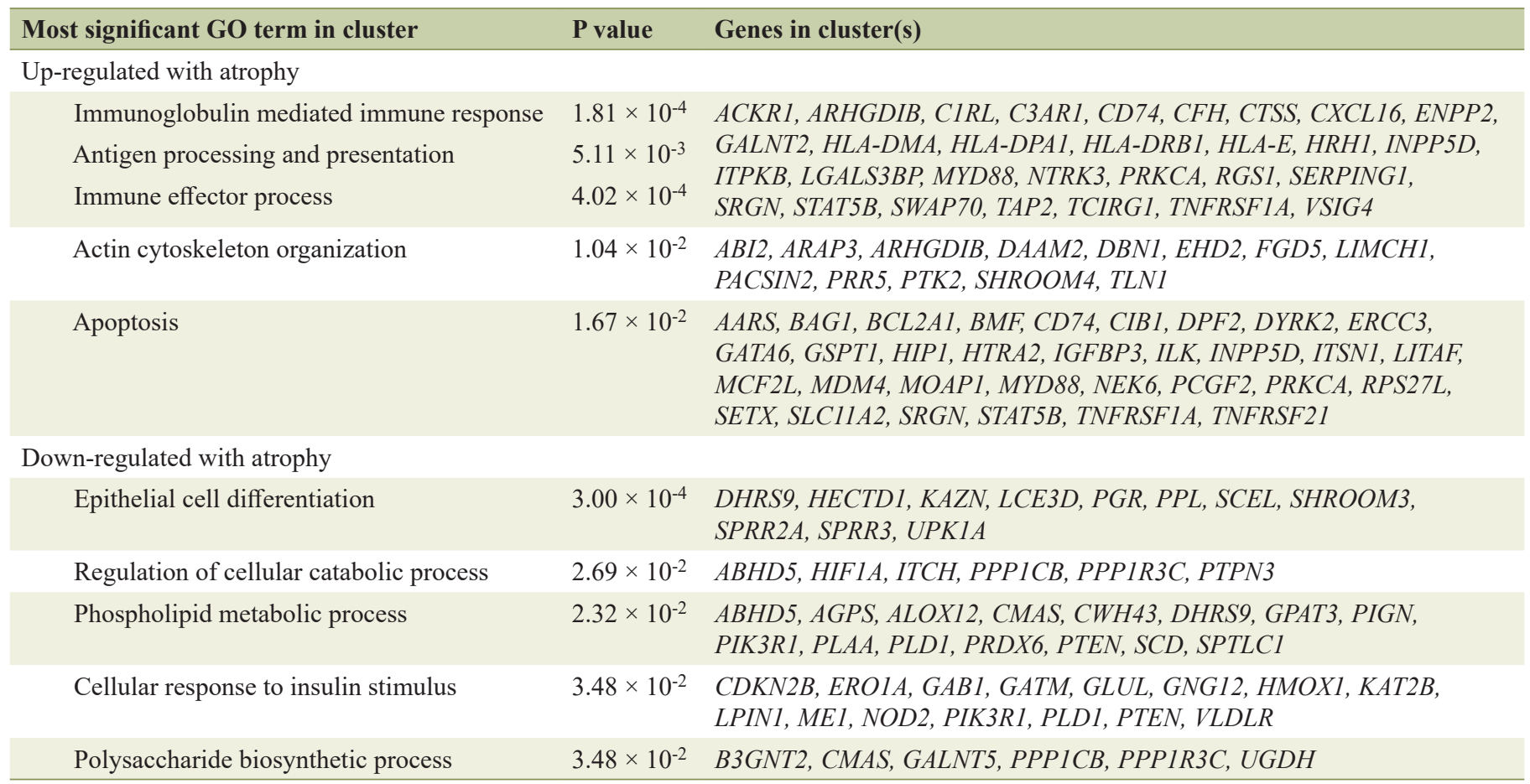

Gene lists from the cross-study comparison presented in Figure 4 were subjected to GO term enrichment analysis as described in "Methods". The table displays results for the five most highly enriched clusters of $\mathrm{GO}$ biological process terms for the up- or down-regulated gene lists and the $\mathrm{P}$ value of the most significant term for each cluster. There was substantial overlap of the genes in the first three up-regulated clusters, so they were grouped together for conciseness. GO: gene ontology.

\section{Discussion}

We compared histology and gene expression at the introitus and labia majora in three cohorts of women who differed based on their atrophic, menopausal and estrogen status. The introitus was selected because it exhibits anatomic/morphologic characteristics of genital atrophy early on, and is often associated with clinical signs and symptoms, particularly dryness and dyspareunia. The labia majora was selected as a site more distal to the vagina because it is composed of hyperhydrated stratified epithelium and had not been studied previously.

From a histological perspective, the changes associated with hypoestrogenicity and atrophy at the introitus appear similar to what has been reported in the upper vagina [3, 20-22]. In addition to a thinner epithelial layer devoid of superficial cells in the atrophic post-menopausal cohort, there was a dramatic loss of rete ridges, or epithelial projections into the papillary dermis (Fig. 2). Rete ridges can be viewed as supporting the structural integrity of the epithelium anchoring it in the connective tissue. Their involution probably contributes to mechanical sensitivity, tissue degradation and reported symptoms of dyspareunia. While there also was a reduction in rete ridge structure in the labia majora, it was more modest, and based on our sample size did not approach significance, suggesting differential site-specific effects of estrogen.

The bioinformatics analysis of the gene expression data focused on global patterns of differentially expressed genes and GO term enrichment analysis to identify biological processes associated with urogenital atrophy. Also, cross-body site and cross-study comparisons and stringent statistical filtering were done to identify key regulated genes. There were profound changes in gene expression in the atrophied introituses of post-menopausal subjects compared to the healthy tissues of pre-menopausal women, with several thousand differentially expressed genes. In contrast, there were far fewer significant gene expression changes in the labia majora of subjects with vaginal atrophy, consistent with the less severe changes in epithelial histology. Overall, the results of hierarchical clustering demonstrated that post-menopausal women on HT, who had ameliorated or escaped atrophic changes, had patterns of gene expression in the introitus and the labia majora much like those in younger pre-menopausal women (Fig. 3).

An earlier investigation studied vaginal gene expression changes in menopausal women between baseline and after 12 weeks systemic ET treatment with estradiol $[11,12]$. The ET reversed atrophic changes in the vagina as indicated by reduced $\mathrm{pH}$ and increased VMI. In a comparative analysis of that study and our data, we found a remarkable close similarity of genes differentially expressed with atrophy in both the vagina (baseline vs. ET treatment) and the introitus (Post-M vs. Pre-M). Of 589 genes meeting statistical filtering, 587 showed the same directional regulation in the two tissues. Quantitative differences in gene expression between the two sites could not be assessed because the data were generated on different GeneChip models and processed differently. However, we demonstrate a 


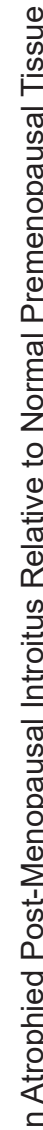

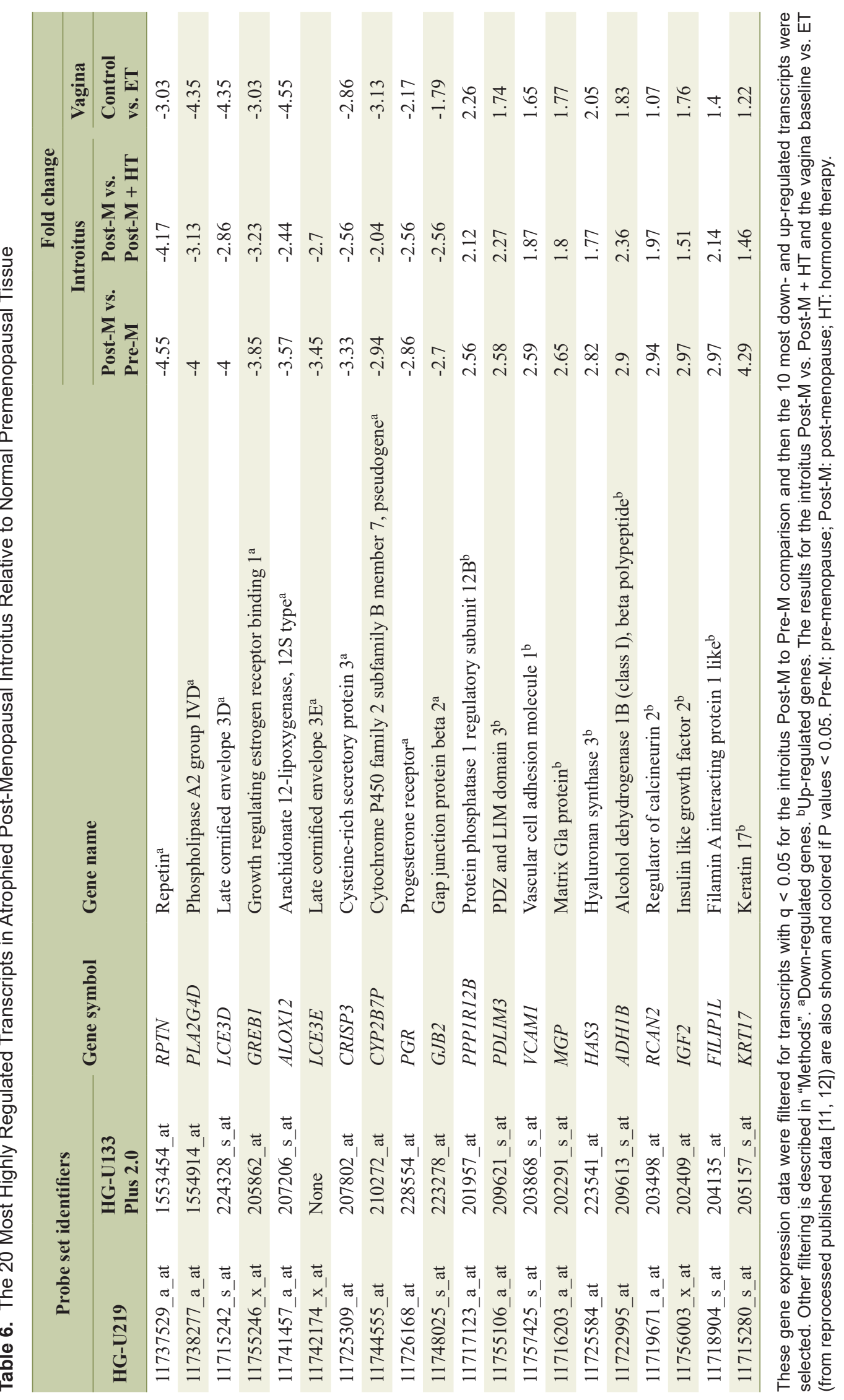


remarkable similarity between two independent gene expression studies done in different laboratories, and that there are many common gene expression changes associated with atrophy in the introitus and the lower third of the vagina. Although both of these studies were small, the high degree of concordance for differentially expressed genes provides evidence that these changes are associated with the atrophic condition.

The GO term enrichment analysis for the introitus Post-M to Pre-M comparison (Table 2) and for the genes identified by the cross-study comparison of atrophy in introitus and vagina (Table 5) showed similarities. Our results also resemble those previously reported for the vagina from the vaginal atrophy treatment study [11, 12] and a study of vaginal dryness [13]. Biological processes relating to a remodeling of tissues were up-regulated with atrophy including "blood vessel morphogenesis", "response to wounding", "cell migration" and "apoptosis". Furthermore, in the introitus and vagina, there was an up-regulation of immune regulation with atrophy, consistent with low level inflammation that is commonly seen in aging tissue [29]. This was also observed in the labia majora. Overall, these up-regulated processes involve genes coding for scores of signaling molecules, including growth factors and chemokines and other inflammatory mediators, extracellular matrix (ECM) components, receptors, components of intracellular signaling pathways and cytoskeleton.

The most significantly down-regulated process in the analyses summarized in Tables 2 and 5 was "epithelial cell differentiation", consistent with epithelial atrophy. This GO term is associated with many significantly down-regulated genes coding for structural components of the differentiating epithelium and factors that regulate differentiation. There also was a general decrease in expression of genes involved in lipid and carbohydrate metabolism, possibly associated with reduced energy production and the reduced glycogen synthesis in the atrophic tissue. Down-regulation of "pyridine nucleotide metabolism" was observed in the introital Post-M to Pre-M comparison (Table 2). That term is associated with a number of down-regulated genes in the pathway for the synthesis of $\mathrm{NAD}^{+}$, a key coenzyme involved in intermediary metabolism. Diminished levels of $\mathrm{NAD}^{+}$have been strongly linked to aging, and it has been suggested that $\mathrm{NAD}^{+}$precursors may be useful interventions to reduce age-related pathologies [30, 31]. The more general GO term "regulation of cellular catabolic process" (Table 5) was associated with some important regulatory genes downregulated with atrophy in both the introitus and vagina. These included abhydrolase domain containing 5 (ABHD5) and hypoxia inducible factor 1 , alpha subunit (HIF1A). ABHD5 plays an essential role in triacylglycerol metabolism necessary for normal epidermal barrier formation [32] and has also been shown to be regulated by 17 -beta-estradiol (E2) in rat vagina [11]. The lower expression of ABHD5 in atrophied tissue is consistent with the general themes of decreased epithelial differentiation and lipid metabolism. Interestingly, knockout or knockdown of HIF $1 A$ has been suggested to accelerate epidermal aging [33], and its decreased expression in atrophied tissue may contribute to the process. A major signaling-related process highlighted in the cross-study comparison was "cellular response to insulin stimulus", which was associated with a number of down-regulated genes. Additional genes related to insulin signaling are described below. Overall, the processes identified suggest that introital and vaginal atrophy involve complex active regulation.

The patterns of expression of collagen-related genes (Table 4) were also consistent with tissue remodeling playing a role in atrophy of the introitus and labia majora. Expression of the major collagens was down-regulated at both sites, although the changes were substantially greater in the introitus. Furthermore, expression of the major collagen genes (COL1A1, COL1A2 and $C O L 3 A 1)$ and $C O L 5 A 2$ were significantly down-regulated at $\mathrm{P}<0.05$ in the vagina (data not shown). In contrast, genes that play a role in collagen fibril formation were expressed at significantly elevated levels in the atrophied introitus (LUM and $D P T)$ and labia majora $(L U M)$. Both $L U M$ and $D P T$ are involved in wound healing [25-27]. Furthermore, expression SFRP2, which modulates WNT pathway signaling [28], was strongly up-regulated with atrophy in the introitus (Table 4) and vagina $(\mathrm{q}<0.05$, data not shown). SFRP2 has been reported to be "dramatically up-regulated" in hypertrophic scars [34], which is also consistent with the general pattern of woundingrelated genes increased in the atrophic tissues. Similar results have been reported from gene expression data comparing older versus younger buttock and forearm skin [35], suggesting that this may be a general pattern in the aging of epithelial tissues.

Statistically stringent cross-comparison of data from the introitus and labia majora identified genes that showed common differential expression in both body sites with atrophy (Table 3). The down-regulated genes were GREB1, EHF, TP63, DIO2, IGF1 and RBM47, and the up-regulated genes were $M F A P 4$ and $C 3$. In our reanalysis of the vaginal data, GREB1, IGF1, RBM47, MFAP4 and C3 showed the same directional regulation as in the introitus and labia majora and were significant at $\mathrm{q}<0.05$. GREB1 is an early response gene in estrogen receptor (ER) signaling and an ER cofactor that may be involved in regulating proliferation of estrogen-responsive tissues $[36,37]$. GREB 1 was among the most down-regulated genes in the atrophied introitus (Table 6). EHF is a member of the epithelial specific expression (ESE) ETS transcription factor family that plays a role in regulating epithelial cell differentiation [38, 39]. Similarly, TP63 is a transcription factor that plays an essential role in the regulation of epithelial morphogenesis and stem cell function [40, 41]. The down-regulation of GREB1, TP63 and EHF may directly contribute to epithelial atrophy in the introitus and labia majora. DIO2 codes for an enzyme that activates thyroid hormone by converting the prohormone thyroxine to triiodothyronine (T3), and the activity of DIO2 controls target tissue levels of T3 [42]. The down-regulation of $\mathrm{DIO} 2$ with atrophy implicates thyroid hormone in the maintenance of both the introitus and labia majora. IGF1 is a member of the insulin growth factor family, which is involved in mediating growth and development and shown to be critically involved in aging $[43,44]$. The role $I G F 1$ in aging appears complex. Although global decreased levels of $I G F 1$ have been associated with increased longevity in diverse species $[43,44], I G F 1$ has also been reported to positively regulate the proteosome and promote beneficial clearance of oxidized proteins in brain [45]. The consistent down-regulation of $I G F 1$ provides evidence of the importance of the insulin pathway in atrophy of the introitus, vagina and labia majora, consistent 
with the GO term enrichment analysis (Table 5). RBM47 was the least down-regulated gene identified in the cross-body site comparison but achieved $\mathrm{q}<0.05$ in both the introitus and vagina. The product of this gene was recently reported to be involved in cytidine (C) to uridine (U) RNA editing, which is a post-transcriptional mechanism regulating gene expression that results in alternative gene products [46]. RBM47 also binds target mRNAs altering their splicing and abundance and acts a suppressor of breast cancer metastasis [47]. The functional consequences of the regulation of $R B M 47$ with atrophy in the introitus, labia majora and vagina are unknown but may contribute to changes in patterns of gene expression.

MFAP4 is an extracellular matrix glycoprotein that plays an important role in elastic fiber organization and belongs to a family of proteins involved in innate immunity [48]. MFAP4 was strongly up-regulated in the introitus and vagina with atrophy and that may indicate a dysregulation of elastic fibrillogenesis. The second gene from the cross-body site analysis that was very strongly up-regulated in both introitus and vagina was C3, a central component of the complement system, which is part of the innate immune response [49]. Complement component 3 a receptor $1(C 3 A R 1)$ and complement factor $\mathrm{H}(\mathrm{CFH})$ genes were also up-regulated in the atrophic introitus and vagina (Table 5), but these did not meet the stringent statistical filtering for the cross-body site comparison. Complement components including $\mathrm{CFH}$ were also reported among the genes up-regulated in post-menopausal women with vaginal dryness [13]. Up-regulation of the expression of complement-related genes is consistent with a general up-regulation of immune response genes in the atrophied tissue noted in other analyses (Tables 2 and 5). Complement is also involved in the removal of apoptotic cells and is linked to aging, including age-related macular degeneration and Alzheimer's disease [49, 50].

The 10 most up- and down-regulated genes in the atrophic introitus filtered for stringent statistical significance are shown in Table 6 along with the vaginal data for comparison. Generally, the regulation of these genes was similar in the introitus and vagina. Among the down-regulated genes in the atrophic introitus were several associated with epithelial differentiation (RPTN, ALOX12, LCE3E, LCE3D and GJB2). The strong down-regulation of these genes is consistent with the marked thinning of the introital epithelium with atrophy (Fig. 2a-c). Since the microarray analysis was done on RNA isolated from biopsy specimens that contain vaginal epithelium as well as dermal connective tissue, and there is decreased thickness of the epithelium with atrophy, it is possible that the measured changes in expression of epithelial differentiation genes were the result of fewer epithelial cells in the samples of atrophic tissue. However, similar changes in gene expression were reported in the study of vaginal dryness [13] in which the samples analyzed were specifically epithelial cells collected using a cytobrush, supporting that these results are meaningful.

Some of the highly regulated genes listed in Table 6 are known to be hormonally regulated, including CRISP3 and the progesterone receptor $(P G R)$ both of which had decreased expression in the atrophic introitus. CRISP 3 is regulated in the menstrual cycle and suggested to play a role in endometrial repair and regeneration [51]. We can speculate that decreased levels may contribute to atrophy. $P G R$ is positively regulated by E2 through complex mechanisms [52] and its diminished expression in the post-M introitus is consistent with decreased circulating estrogen levels (Table 1). In contrast, IGF2 showed very strong up-regulation in the atrophied Post-M introitus and vagina. This increased expression may be related to increased circulating follicle-stimulating hormone (FSH), which is elevated in menopause and sometimes used as a biomarker for staging menopausal progression [53]. FSH has been reported to positively regulate $I G F 2$ expression in human granulosa cells [54]. The strong regulation of IGF2 with introital and vaginal atrophy is consistent with the data discussed earlier pointing to the involvement of insulin pathway signaling in introital and vaginal atrophy. Genes on this pathway are both up- (e.g. $I G F 2$ ) and down-regulated (e.g. $I G F 1)$ with atrophy. The strongest up-regulated gene in the post-M to pre-M introital comparison was KRT17. However, KRT17 expression was not significantly different in the Post-M to Post-M + HT introitus and baseline vs. estrogen-treated vaginal comparisons. KRT17 codes for an unusual intermediate filament that has immunomodulatory activity and is up-regulated in inflammatory conditions such as psoriasis and epithelial wound healing [55, 56], which has been a recurring theme in this "Discussion".

In summary, post-menopausal atrophy of the introitus and to a lesser extent the labia majora were associated with marked changes in epithelial histology and patterns of gene expression. The gene expression changes were consistent with epithelial involution, remodeling of the dermal matrix, low level chronic inflammation and decreases in metabolic processes.

\section{Potential clinical value}

While the use of topical vaginal estrogen has been shown to be efficacious in preventing/treating GSM, the commonly prescribed mode of administration is to use an applicator that delivers the estrogen to the proximal or upper portion of the vagina. This study supports the potential utility of treatments that are designed to target the distal vagina (i.e. the introitus) as well as the development of treatments that are more targeted toward specific pathways associated with atrophy.

\section{Acknowledgments}

We acknowledge Robyn Swartz for her assistance with microscopy and Rachel Adams for reprocessing published microarray data.

\section{Financial Disclosure}

The Procter and Gamble Company.

\section{Conflict of Interest}

MA Freedman and KB Sharma are consultants for The Procter \& Gamble Company and received funding from Procter \& 
Gamble for this study. RL Binder, MA Farage, Y Wang, C Combs, D Moore, JP Tiesman, CC Bascom, RJ Isfort and R Warren are current or former employees of Procter \& Gamble.

\section{Informed Consent}

Written informed consent was obtained from all subjects prior to any study related procedure.

\section{Author Contributions}

RL Binder: study design, data analysis and interpretation, drafting and revising manuscript. MA Freedman: study conception and design, recruiting subjects, clinical procedures, manuscript editing. KB Sharma: directed histopathology and clinical chemistry analysis. MA Farage: study design, manuscript editing. Y Wang: statistical analysis and data summary. C Combs: clinical sample processing, laboratory data generation and summary. D Moore: image analysis. JP Tiesman: study design, supervised laboratory data generation and summary. CC Bascom: study conception and design, review of manuscript. RJ Isfort: study conception, review of manuscript. R Warren: study conception and design, data analysis and interpretation, drafting and revising manuscript.

\section{References}

1. Pandit L, Ouslander JG. Postmenopausal vaginal atrophy and atrophic vaginitis. Am J Med Sci. 1997;314(4):228231.

2. Freedman MA. Vaginal $\mathrm{pH}$, estrogen and genital atrophy. Menopause Manage. 2008;17(4):9-13.

3. Brizzolara S, Killeen J, Severino R. Vaginal $\mathrm{pH}$ and parabasal cells in postmenopausal women. Obstet Gynecol. 1999;94(5 Pt 1):700-703.

4. Bercovici B, Uretzki G, Palti Y. The effects of estrogens on cytology and vascularization of the vaginal epithelium in climacteric women. Am J Obstet Gynecol. 1972;113(1):98-103.

5. Brotman RM, Shardell MD, Gajer P, Fadrosh D, Chang K, Silver MI, Viscidi RP, et al. Association between the vaginal microbiota, menopause status, and signs of vulvovaginal atrophy. Menopause. 2014;21(5):450-458.

6. Gorodeski GI. Effects of estrogen on proton secretion via the apical membrane in vaginal-ectocervical epithelial cells of postmenopausal women. Menopause. 2005;12(6):679-684.

7. Portman DJ, Gass ML, Vulvovaginal Atrophy Terminology Consensus Conference P. Genitourinary syndrome of menopause: new terminology for vulvovaginal atrophy from the International Society for the Study of Women's Sexual Health and the North American Menopause Society. Menopause. 2014;21(10):1063-1068.

8. Kingsberg SA, Wysocki S, Magnus L, Krychman ML. Vulvar and vaginal atrophy in postmenopausal women: findings from the REVIVE (REal Women's VIews of Treatment Options for Menopausal Vaginal ChangEs) survey. J Sex Med. 2013;10(7):1790-1799.

9. Simon JA, Kokot-Kierepa M, Goldstein J, Nappi RE. Vaginal health in the United States: results from the Vaginal Health: Insights, Views \& Attitudes survey. Menopause. 2013;20(10):1043-1048.

10. Huang AJ, Luft J, Grady D, Kuppermann M. The dayto-day impact of urogenital aging: perspectives from racially/ethnically diverse women. J Gen Intern Med. 2010;25(1):45-51.

11. Jelinsky SA, Choe SE, Crabtree JS, Cotreau MM, Wilson E, Saraf K, Dorner AJ, et al. Molecular analysis of the vaginal response to estrogens in the ovariectomized rat and postmenopausal woman. BMC Med Genomics. 2008;1:27.

12. Cotreau MM, Chennathukuzhi VM, Harris HA, Han L, Dorner AJ, Apseloff G, Varadarajan U, et al. A study of 17beta-estradiol-regulated genes in the vagina of postmenopausal women with vaginal atrophy. Maturitas. 2007;58(4):366-376.

13. Hummelen R, Macklaim JM, Bisanz JE, Hammond JA, McMillan A, Vongsa R, Koenig D, et al. Vaginal microbiome and epithelial gene array in post-menopausal women with moderate to severe dryness. PLoS One. 2011;6(11):e26602.

14. Kallak TK, Baumgart J, Nilsson K, Akerud H, Poromaa IS, Stavreus-Evers A. Vaginal Gene Expression During Treatment With Aromatase Inhibitors. Clin Breast Cancer. 2015;15(6):527-535 e522.

15. GEO Database. Available at: http://www.ncbi.nlm.nih. gov/geo.

16. Boca SM, Leek JT. A direct approach to estimating false discovery rates conditional on covariates. PeerJ. 2018;6:e6035.

17. Gene Ontology Consortium. Available at: http://geneontology.org.

18. Huang da W, Sherman BT, Lempicki RA. Bioinformatics enrichment tools: paths toward the comprehensive functional analysis of large gene lists. Nucleic Acids Res. 2009;37(1):1-13.

19. Huang da W, Sherman BT, Lempicki RA. Systematic and integrative analysis of large gene lists using DAVID bioinformatics resources. Nat Protoc. 2009;4(1):44-57.

20. Nilsson K, Risberg B, Heimer G. The vaginal epithelium in the postmenopause - cytology, histology and $\mathrm{pH}$ as methods of assessment. Maturitas. 1995;21(1):51-56.

21. Blakeman PJ, Hilton P, Bulmer JN. Cellular proliferation in the female lower urinary tract with reference to oestrogen status. BJOG. 2001;108(8):813-816.

22. Kallak TK, Baumgart J, Goransson E, Nilsson K, Poromaa IS, Stavreus-Evers A. Aromatase inhibitors affect vaginal proliferation and steroid hormone receptors. Menopause. 2014;21(4):383-390.

23. Herchenhan A, Uhlenbrock F, Eliasson P, Weis M, Eyre D, Kadler KE, Magnusson SP, et al. Lysyl oxidase activity is required for ordered collagen fibrillogenesis by tendon cells. J Biol Chem. 2015;290(26):16440-16450.

24. Risteli M, Ruotsalainen H, Salo AM, Sormunen R, Sipila L, Baker NL, Lamande SR, et al. Reduction of lysyl hy- 
droxylase 3 causes deleterious changes in the deposition and organization of extracellular matrix. J Biol Chem. 2009;284(41):28204-28211.

25. Okamoto O, Fujiwara S. Dermatopontin, a novel player in the biology of the extracellular matrix. Connect Tissue Res. 2006;47(4):177-189.

26. Yeh JT, Yeh LK, Jung SM, Chang TJ, Wu HH, Shiu TF, Liu CY, et al. Impaired skin wound healing in lumicannull mice. Br J Dermatol. 2010;163(6):1174-1180.

27. Liu XJ, Kong FZ, Wang YH, Zheng JH, Wan WD, Deng CL, Mao GY, et al. Lumican Accelerates Wound Healing by Enhancing alpha2beta1 Integrin-Mediated Fibroblast Contractility. PLoS One. 2013;8(6):e67124.

28. Jones SE, Jomary C. Secreted Frizzled-related proteins: searching for relationships and patterns. Bioessays. 2002;24(9):811-820.

29. Franceschi C, Campisi J. Chronic inflammation (inflammaging) and its potential contribution to age-associated diseases. J Gerontol A Biol Sci Med Sci. 2014;69(Suppl 1):S4-9.

30. Verdin E. NAD $(+)$ in aging, metabolism, and neurodegeneration. Science. 2015;350(6265):1208-1213.

31. Kaeberlein M, Rabinovitch PS, Martin GM. Healthy aging: The ultimate preventative medicine. Science. 2015;350(6265):1191-1193.

32. Radner FP, Fischer J. The important role of epidermal triacylglycerol metabolism for maintenance of the skin permeability barrier function. Biochim Biophys Acta. 2014;1841(3):409-415.

33. Rezvani HR, Ali N, Serrano-Sanchez M, Dubus P, Varon C, Ged C, Pain C, et al. Loss of epidermal hypoxia-inducible factor-1alpha accelerates epidermal aging and affects re-epithelialization in human and mouse. J Cell Sci. 2011;124(Pt 24):4172-4183.

34. Chen L, Wang Z, Li S, Zhao G, Tian M, Sun Z. SFRP2 and slug contribute to cellular resistance to apoptosis in hypertrophic scars. PLoS One. 2012;7(12):e50229.

35. Robinson MK, Binder RL, Griffiths CE. Genomic-driven insights into changes in aging skin. J Drugs Dermatol. 2009;8(7 Suppl):s8-11.

36. Mohammed H, D'Santos C, Serandour AA, Ali HR, Brown GD, Atkins A, Rueda OM, et al. Endogenous purification reveals GREB1 as a key estrogen receptor regulatory factor. Cell Rep. 2013;3(2):342-349.

37. Ghosh MG, Thompson DA, Weigel RJ. PDZK1 and GREB1 are estrogen-regulated genes expressed in hormone-responsive breast cancer. Cancer Res. 2000;60(22):6367-6375.

38. Tugores A, Le J, Sorokina I, Snijders AJ, Duyao M, Reddy PS, Carlee L, et al. The epithelium-specific ETS protein EHF/ESE-3 is a context-dependent transcriptional repressor downstream of MAPK signaling cascades. J Biol Chem. 2001;276(23):20397-20406.

39. Stephens DN, Klein RH, Salmans ML, Gordon W, Ho $\mathrm{H}$, Andersen B. The Ets transcription factor EHF as a regulator of cornea epithelial cell identity. J Biol Chem. 2013;288(48):34304-34324.

40. Senoo M, Pinto F, Crum CP, McKeon F. p63 Is essential for the proliferative potential of stem cells in stratified epithelia. Cell. 2007;129(3):523-536.
41. Truong AB, Kretz M, Ridky TW, Kimmel R, Khavari PA. p63 regulates proliferation and differentiation of developmentally mature keratinocytes. Genes Dev. 2006;20(22):3185-3197.

42. Williams GR, Bassett JH. Deiodinases: the balance of thyroid hormone: local control of thyroid hormone action: role of type 2 deiodinase. J Endocrinol. 2011;209(3):261-272.

43. Kenyon CJ. The genetics of ageing. Nature. 2010;464(7288):504-512.

44. Murphy T, Thuret S. The systemic milieu as a mediator of dietary influence on stem cell function during ageing. Ageing Res Rev. 2015;19:53-64.

45. Crowe E, Sell C, Thomas JD, Johannes GJ, Torres C. Activation of proteasome by insulin-like growth factor-I may enhance clearance of oxidized proteins in the brain. Mech Ageing Dev. 2009;130(11-12):793-800.

46. Fossat N, Tourle K, Radziewic T, Barratt K, Liebhold D, Studdert JB, Power M, et al. C to U RNA editing mediated by APOBEC1 requires RNA-binding protein RBM47. EMBO Rep. 2014;15(8):903-910.

47. Vanharanta S, Marney CB, Shu W, Valiente M, Zou Y, Mele A, Darnell RB, et al. Loss of the multifunctional RNA-binding protein RBM47 as a source of selectable metastatic traits in breast cancer. Elife. 2014;3:e02734.

48. Pilecki B, Holm AT, Schlosser A, Moeller JB, Wohl AP, Zuk AV, Heumuller SE, et al. Characterization of Microfibrillar-associated Protein 4 (MFAP4) as a Tropoelastinand Fibrillin-binding Protein Involved in Elastic Fiber Formation. J Biol Chem. 2016;291(3):1103-1114.

49. Ricklin D, Hajishengallis G, Yang K, Lambris JD. Complement: a key system for immune surveillance and homeostasis. Nat Immunol. 2010;11(9):785-797.

50. Patel N, Adewoyin T, Chong NV. Age-related macular degeneration: a perspective on genetic studies. Eye (Lond). 2008;22(6):768-776.

51. Evans J, D'Sylva R, Volpert M, Jamsai D, Merriner DJ, Nie G, Salamonsen LA, et al. Endometrial CRISP3 is regulated throughout the mouse estrous and human menstrual cycle and facilitates adhesion and proliferation of endometrial epithelial cells. Biol Reprod. 2015;92(4):99.

52. Vazquez-Martinez ER, Mendoza-Garces L, Vergara-Castaneda E, Cerbon M. Epigenetic regulation of Progesterone Receptor isoforms: from classical models to the sexual brain. Mol Cell Endocrinol. 2014;392(1-2):115-124.

53. Hale GE, Robertson DM, Burger HG. The perimenopausal woman: endocrinology and management. J Steroid Biochem Mol Biol. 2014;142:121-131.

54. Baumgarten SC, Convissar SM, Zamah AM, Fierro MA, Winston NJ, Scoccia B, Stocco C. FSH regulates IGF-2 expression in human granulosa cells in an AKT-dependent manner. J Clin Endocrinol Metab. 2015;100(8):E10461055.

55. Depianto D, Kerns ML, Dlugosz AA, Coulombe PA. Keratin 17 promotes epithelial proliferation and tumor growth by polarizing the immune response in skin. Nat Genet. 2010;42(10):910-914.

56. Kim S, Wong P, Coulombe PA. A keratin cytoskeletal protein regulates protein synthesis and epithelial cell growth. Nature. 2006;441(7091):362-365. 